\title{
Hadron Physics with Strange and Charm Quarks - The PANDA Experiment.
}

\author{
Olaf N. Hartmann ${ }^{* \dagger}$ \\ Stefan-Meyer-Institut für Subatomare Physik, Österreichische Akademie der Wissenschaften, \\ Boltzmanngasse 3, 1090 Vienna, Austria \\ E-mail: olaf.hartmann@oeaw.ac.at
}

PANDA is a new large-scale experiment in hadron physics which will be built together with the new accelerator complex FAIR in Darmstadt, Germany. It will use annihilations of antiprotons to study hadron properties in the strange and charm quark sector. This contribution describes the facility, selected physics topics and the detector concept.

The Xth Nicola Cabibbo International Conference on Heavy Quarks and Leptons, October 11-15, 2010

Frascati (Rome) Italy

\footnotetext{
* Speaker.

${ }^{\dagger}$ On behalf of the PANDA Collaboration.
} 


\section{FAIR and the HESR}

At the site of the GSI Helmholtz-Centre for Heavy Ion Research [1] in Darmstadt, Germany, a new accelerator complex called FAIR (Facility for Antiproton and Ion Research, [2]) will be constructed in the upcoming years. In october 2010 the international convention was signed by nine partner countries and thus FAIR was legally founded. Fig. 11 shows the layout of the facility with the new parts in red; the blue components are existing accelerators of the GSI.

As a central pillar, FAIR will offer antiproton beams of high quality and intensity. A primary proton beam of $30 \mathrm{GeV}$ from the SIS100 will be used to produce antiprotons on an appropriate production target. The antiprotons will be collected and cooled and transfered at a fixed momentum of $3.5 \mathrm{GeV} / \mathrm{c}$ into the HESR. The High Energy Storage and Synchrotron Ring HESR is a dedicated accelerator which stores the antiprotons (up to $10^{11}$ ) and accelerates/decelerates them within a momentum range from 1.5 to $15 \mathrm{GeV} / \mathrm{c}$. In the first phase of its operation, the HESR serves exclusively the PANDA experiment, a spectrometer with an internal target. The HESR is designed to work in two operation modes:

- High resolution mode: the momentum resolution reaches $\frac{\delta_{p}}{p}=2 \cdot 10^{-5}$ at a luminosity of $2 \cdot 10^{31} \mathrm{~cm}^{-2} \mathrm{~s}^{-1}$.

- High luminosity mode: a luminosity of $2 \cdot 10^{32} \mathrm{~cm}^{-2} \mathrm{~s}^{-1}$ is provided at a momentum resolution of $\frac{\delta_{p}}{p} \sim 10^{-4}$

Up to a beam momentum of $9 \mathrm{GeV} / \mathrm{c}$ electron cooling is available, as well as stochastic cooling over the full momentum range.

In the so-called start version of FAIR, the RESR, a ring in between the collector ring and the HESR, is not included. As a consequence, the HESR has also to do antiproton accumulation which limits the maximum antiproton intensity to $10^{10}$ stored particles.

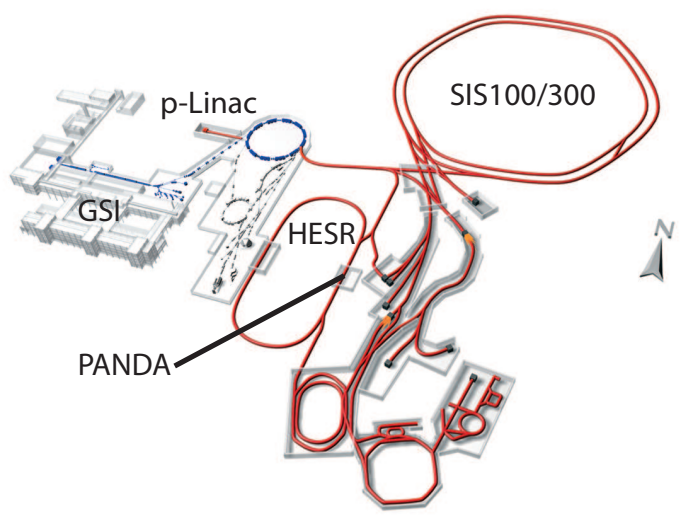

Figure 1: Layout of FAIR. The existing GSI is also show, with its accelerators in blue. The red lines correspond to the new FAIR accelerators and storage rings. The position of the PANDA experiment in the HESR is marked. 


\section{The PANDA Physics}

The momentum range of the HESR allows to access particles in the mass region of strange and charm quarks $\left(2.2 \mathrm{GeV} / \mathrm{c}^{2}<\sqrt{s}<5.5 \mathrm{GeV} / \mathrm{c}^{2}\right)$. At these energies, the coupling constant of the strong interaction $\alpha_{s}$ is already too large to allow for perturbative treatment of QCD, the relevant degrees of freedom are hadrons. The PANDA physics program covers

- Hadron Spectroscopy

Charmonium and open charm mesons, search for exotics like glueballs and hybrids, multiquark states, baryons, ...

- Hadron properties in nuclear matter

How does the mass of hadrons come about?

- Nonperturbative dynamics

Transition from parton picture to hadrons

- Single and double hypernuclei

Hyperon-nucleon and hyperon-hyperon interaction

- Nucleon Structure

timelike electromagnetic form factors, transverse parton distributions, ...

- Electroweak physics

CP-violation in open charm systems, ...

For the first two items, examples will be presented in the following subsections. A more complete description of the program as well as more details on the following can be found in [4].

\subsection{Hadron Spectroscopy}

The search for exotic states like glueballs and hybrids is a key mission of PANDA. Whereas all states with quantum numbers allowed in the q $\bar{q}$ system can be formed directly in the $\bar{p} p$ annihilation, hybrid and glueball states are predicted to have also exotic quantum numbers $\left(J^{P C}=1^{-+}\right.$ and $0^{+-}, 2^{+-}$("oddballs"), respectively). They should have masses in the range between 4 and $5 \mathrm{GeV} / \mathrm{c}^{2}$ and widths smaller than $50 \mathrm{MeV}$ [5], hence accessible in production experiments with PANDA.

The possibility to directly form all states in the charmonium (cc) spectrum combined with the excellent momentum resolution of the HESR which translates into up to $50 \mathrm{keV}$ resolution in $E_{C M}$, makes PANDA an ideal tool to study the complete spectrum in detail, determining widths and masses with high prescision.

Experiments at B-factories like BaBar@SLAC, BELLE@KEK-B, also CDF, D0, CLEO have reported the discovery of many new states, usually quoted as $X Y Z$ states, which still have lots of undetermined and not understood properties. For instance, despite of lying above the D $\overline{\mathrm{D}}$ threshold, the $Y$ (4260) [6] does not decay into open charm but rather in final states containing a $J / \psi$. Using experimental information from BaBar and CLEO, like the cross section and the $\pi^{+} \pi^{-}$ 


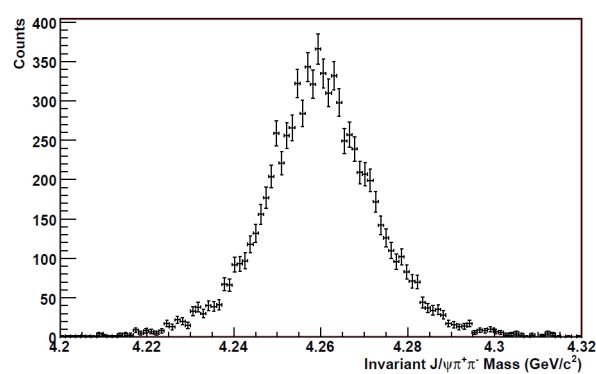

Figure 2: The $Y(4260)$ in the $J / \psi \pi^{+} \pi^{-}$invariant mass, reconstructed in the PANDA monte carlo.

mass spectrum shape, the reconstruction of $Y(4260)$ in the $J / \psi \pi^{+} \pi^{-}$channel has been studied in the PANDA monte carlo (see [4]), including studies of the main background which comes from $\overline{\mathrm{p}} \rightarrow \pi^{+} \pi^{-} \pi^{+} \pi^{-}$. A rate of $100 \mathrm{~d}^{-1}$ and a signal-over-background-rate of 2 has been estimated, with a reconstruction efficiency of 0.32 . The resulting spectrum is shown in Fig. 2. Furthermore, in the open charm sector new narrow states called $\mathrm{D}_{s 0}^{*}(2317)$ and $\mathrm{D}_{s}^{*}(2460)$ have been reported (see e.g. (7). These states, close to the $\mathrm{DK}$ and $\mathrm{D}^{*} \mathrm{~K}$ threshold, respectively, do not quite agree with quark model predictions which favour higher masses. Concerning e.g. the width of $\mathrm{D}_{s 0}^{*}(2317)$, various theoretical predictions ranging from 6 to $180 \mathrm{keV}$ are on the market. A possible reaction channel to be studied at PANDA is

$$
\overline{\mathrm{p}} \mathrm{p} \rightarrow \mathrm{D}_{s}^{ \pm} \mathrm{D}_{s 0}^{*}(2317)^{\mp} .
$$

Making use of the very good momentum resolution of the HESR beam a threshold scan (excitation function for mass and width) can be used to determine the width experimentally.

The cross sections into baryon-antibaryon channels are comparable to those into mesons. For the $S=-2$ and $S=-2$ baryons there's little known experimentally so far, many resonance states are predicted (e.g. [8]). Thus, baryon spectroscopy has discovery potential, and can be perfomed at PANDA, up to the charmed baryons. As an example, the study of the reaction

$$
\overline{\mathrm{p}} \rightarrow \Xi^{+} \Xi^{-} \pi^{0}
$$

which involves the double strange $\Xi$ baryon has been evaluated. A reconstruction of the $\Xi^{-} \rightarrow \Lambda \pi^{-}\left(\Lambda \rightarrow \mathrm{p} \pi^{-}\right)$is necessary, and the PANDA monte carlo shows that a $\Xi^{-} \pi^{0}$ mass can be determined with $\sim 4 \mathrm{MeV}$ resolution and a decent efficiency. However, the identification of specific $\Xi$ resonances requires partial wave analysis.

\subsection{Hadron Properties in Nuclear Matter}

An unique feature of PANDA is the possibility to perform antiproton annihilations on nuclear targets. Such reactions can be used to study the properties of hadrons in nuclear matter. A lot of effort has been done in this respect in the light and strange quark sector (see e.g. [9, 10]). Following [11], also in the case of normal nuclear matter density at temperature zero, i.e. with $\overline{\mathrm{p}}$ beams, sizeable effects are predicted which can manifest themselves as changes in mass and width of hadrons in medium with respect to their vacuum properties. At PANDA these studies can be extended into the charmed hadron mass range which was not exploited before. For instance, 
subthreshold production of $\mathrm{D}^{ \pm}$can be studied. In the case of dropping $\mathrm{D}$ masses a certain yield should be detected below the vacuum production threshold. Also the formation of (quasi)bound states might be an effect, like recently calculated in [12] for $\mathrm{D}^{0}$-nucleus bound states. Such states may be accessible in a reaction like

$$
\overline{\mathrm{p}}+A_{Z} \rightarrow \mathrm{D}^{*+}+X ; \quad \mathrm{D}^{*+}+A_{Z} \rightarrow \pi^{+}+\left\{A_{Z}-\mathrm{D}^{0}\right\}_{\text {bound }} .
$$

The evaluation of the dissociation cross section of the $J / \psi$ in the nuclear environment can also be done in $\overline{\mathrm{p}}$ induced reactions at PANDA. Measurable quantities are the $J / \psi$ yield in the elementary reaction and in nuclei by scanning around the $J / \psi$ resonance. From a monte carlo model the probability to form a $J / \psi$ in a nucleus can be estimated, including density effects and the Fermi momenta of the nucleons. The dissociation cross section comes as 1 minus the survival probability of a formed $J / \psi$ folded with the travelled path in the nucleus. Informations about the dissociation of $J / \psi$ are relevant in the interpretation of the $J / \psi$ suppression in ultrarelativitistic heavy ion collisions as one of the signals of quark-gluon-plasma (QGP) formation (reported e.g. in [13]).

\section{The PANDA Detector}

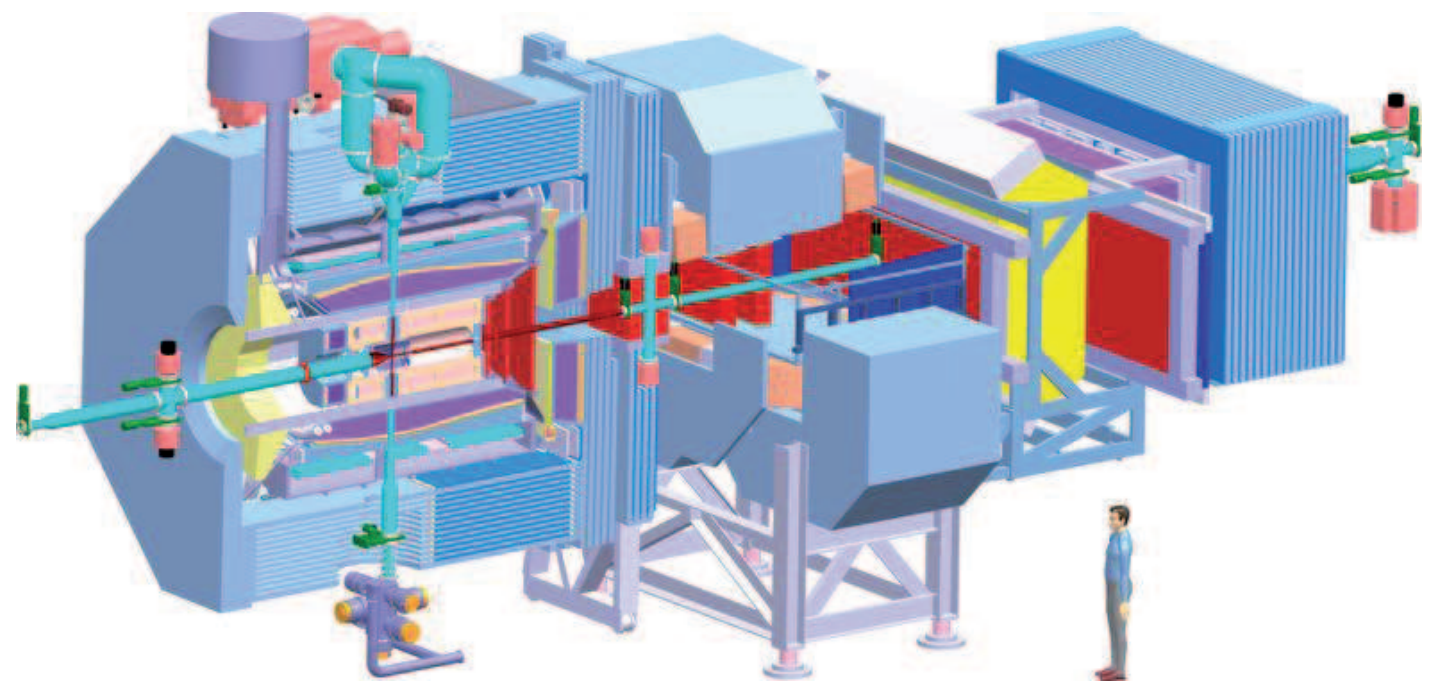

Figure 3: The PANDA detector. For a description of the components see text.

The PANDA detector layout is shown in Fig. 3 . The $\overline{\mathrm{p}}$ beam is entering from the left site, and beam particles which did not interact follow the beam pipe throughout the system for eventually another cycle in the HESR. Since PANDA is mounted on a storage ring it uses an internal target which will be realized by a target pipe which has a joint cross with the beampipe at the interaction point. Beam- and targetpipe have a $20 \mathrm{~mm}$ diameter at the crossing. The target beam, which in the case of a hydrogen (proton) target will be made out of a high-density cluster gas jet or frozen hydrogen pellets, comes from the top of the setup. Heavier gases can be used a well in this scheme, and wires/thin foils for nuclear target are envisaged, too. The target spectrometer part of PANDA, around the interaction point, is embedded in a superconducting solenoid with provides a $2 \mathrm{~T}$ magnetic field along the beam axis. More downstream a dipole magnet with up to $2 \mathrm{Tm}$ bending power 
is placed with the PANDA forward spectrometer.

The innermost dectector in the target spectrometer is the Microvertex detector (MVD), which uses silicon pixel/silicon strip techniques to achieve a spatial resolution of $\sim 50 \mu \mathrm{m}$, important for the detection of secondary vertices from short lived particles $\left(\right.$ like $\left.\mathrm{D}_{s}\right)$. Further out, the central tracking detector will measure charged particle momenta (with $\sim 1 \%$ resolution), $\frac{d E}{d x}$ and multiple vertices. This detector will be realized either by straw tubes or a time projection chamber with GEM-readout. The tracking information for the forward angles will be provideded by a stack of GEM foils. For particle identification, a cherenkov detector using the DIRC technique with a barrel and a forward disc is placed, as well as an electromagnetic calorimeter (EMC). The DIRC is able to separate charged pions and kaons as well as protons with momenta above $1 \mathrm{GeV} / \mathrm{c}$. The EMC is made out of PWO crystals, provides an energy resolution of $\frac{\sigma_{E}}{\sqrt{E}}<2 \%$ and measures photons from $1 \mathrm{MeV}$ to $10 \mathrm{GeV}$ energy. It has a barrel part and a backward and a forward endcap. Finally, the iron yoke of the solenoid is instrumented with a muon detection system. For the hypernuclear physics the backward parts of the target spectrometer are replaced by a different setup.

The forward spectrometer employs various tracking stations, i.a. made with straw tubes, an electromagnetic calorimeter $\left(\frac{\sigma_{E}}{\sqrt{E}} \approx 4 \%\right)$ and a hadron calorimeter. Additional particle identification detectors like a RICH-type cherenkov detector are optional.

The technical design reports (TDR) for the magnets and the EMC are already submitted and approved, and the remaining TDRs will follow in the next years. Concerning the build-up of PANDA, the civil contruction plans of FAIR foresee the availability of the appropriate buildings for the middle of 2016. A pre-assembly of nearly the complete setup of PANDA will be done before. This procedure hopefully points out potential problems and helps to solve them before the final setup at FAIR-HESR.

\section{References}

[1] http://www.gsi.de

[2] http://www.fair-center.org

[3] http://www-panda.gsi.de

[4] Physics Performance Report for PANDA, The PANDA Collaboration, 2009, arxiv:0903.3905v1

[5] G. Bali, Eur.Phys.Journ. A 19 (2004) 1

[6] B. Aubert et al., Phys.Rev.Lett. 94 (2005) 142001

[7] B. Aubert et al., Phys.Rev.D 74 (2006) 032007, [arXiv:hep-ex/0604030v2]

[8] Löring and Metsch, Eur.Phys.Journ. A 10 (2001) 447

[9] R. Hayano and T. Hatsuda, Rev. Mod. Phys. 82 (2010) 2950

[10] U. Mosel, S. Leupold, V. Metag, arXiv:1006.5822v1 [nucl-th]

[11] S. Klimt, M. Lutz, W. Weise, Phys.Lett.B 249 (1990) 386

[12] C. García Recio, J. Nieves, L. Tolos, Phys.Lett. B 690 (2010) 369

[13] http://newstate-matter.web.cern.ch/newstate-matter/ 